\section{In vivo analysis of heat-shock-protein-70 induction following pulsed laser irradiation in a transgenic reporter mouse}

\author{
Caitlin E. O'Connell-Rodwell, ${ }^{\text {a,c }}$ Mark A. Mackanos, ${ }^{a}$ \\ Dmitri Simanovskii, ${ }^{b}$ Yu-An Cao, ${ }^{a}$ \\ Michael H. Bachmann, ${ }^{a}$ H. Alan Schwettman, ${ }^{b}$ and \\ Christopher H. Contag ${ }^{a, *}$ \\ ${ }^{\text {a }}$ Stanford School of Medicine, Stanford, California 94305 \\ ${ }^{\mathrm{b}}$ Stanford University, W.W. Hansen Experimental \\ Physics Laboratory, Stanford, California 94305 \\ 'Stanford University, Department of Otolaryngology, \\ 301 Welsh Rd., Stanford, California 94305
}

\begin{abstract}
Induction of heat shock protein (Hsp) expression appears to correlate with a cytoprotective effect in cultured cells and with improved healing of damaged tissues in animal models and in humans. This family of proteins can also serve as indicators of thermal stress in cases of burn injury or surgical procedures that produce heat. Thus, a rapid in vivo readout for induction of Hsp transcription would facilitate studies of Hsp genes and their encoded proteins as mediators of therapeutic effects and as reporters of thermal damage to tissues. We created a transgenic reporter mouse where expression of luciferase is controlled by the regulatory region of the inducible $70 \mathrm{kDa} \mathrm{Hsp}$, and assessed activation of Hsp70 transcription in live animals in response to rapid, high temperature stresses using in vivo bioluminescence imaging (BLI). This model can be used to noninvasively reveal levels of Hsp70 transcription in living tissues, and has utility in studies of the predictive and protective effects of Hsp70 expression, and of various stress responses in tissues.

(C) 2008 Society of Photo-Optical Instrumentation Engineers. [DOI: $10.1117 / 1.2904665]$
\end{abstract}

Keywords: laser-tissue interactions; luciferase; molecular imaging; heat-shock-protein-70; thermal stress.

Paper 07350LR received Aug. 24, 2007; revised manuscript received Nov. 8, 2007; accepted for publication Dec. 21, 2007; published online May 6, 2008.

\section{Introduction}

The heat shock response of cells is thought to have a role in ameliorating cellular damage and preventing programmed cell death in thermal and other stress environments. ${ }^{1}$ The protective effects of heat shock proteins (Hsps) are likely mediated by the ability of these proteins to function as molecular chaperones, preventing inappropriate protein aggregation and facilitating transport of immature proteins to target organelles for packing, degradation, or repair. Increased expression of

*Address all correspondence to: Christopher H. Contag, Department of Pediatrics, Stanford School of Medicine, Clark Center E-150, 318 Campus Drive, Stanford, CA 94035-5427. Tel: 650-725-8781; Fax: 650-498-7723; E-mail:ccontag@stanford.edu.
Hsp70 in response to temperature slightly above physiological temperatures (e.g., $42^{\circ} \mathrm{C}$ ), for periods of several minutes has been studied as a potential treatment for indications such as ischemic heart disease, diabetes, neurodegeneration, cancer, and thermal damage. ${ }^{1,2}$ Hsp70 expression can be induced at the level of transcription in all cell and tissue types that have been examined, and significant intraspecies sequence similarity suggests that it has been conserved through evolution.

Ablation techniques that employ lasers for tissue removal or remodeling can result in very high temperatures for extremely short periods of time, and levels of Hsp expression in adjacent tissues may correlate with healing or may predict the extent of tissue damage. Previous studies have shown that Hsps serve as strong predictors for cancer cell survival and resistance following laser cancer therapy. ${ }^{1,3}$ Understanding the patterns of Hsp transcription in tissues surrounding ablation sites will reveal the nature of the accompanying stress and may aid in the development of more effective tissue ablation and regeneration strategies. Ferrando et al. first showed induction of $\mathrm{Hsp} 70$ in cells using a $\mathrm{CO}_{2}$ laser with a one second pulse duration. ${ }^{4}$ Others have quantified the expression of Hsp70 using bioluminescent reporters in cells and "raft cultures," a surrogate tissue model, ${ }^{5-7}$ after exposure to thermal stresses.

The effects of high temperature exposure for extremely short durations during irradiation may differ significantly given that the shock is determined by the time of energy deposition (e.g., laser pulse duration), and the heat dissipation time (thermal diffusion time), which can greatly exceed the energy deposition time. In situations where the energy deposition time is less than the thermal confinement time (the time associated with heat remaining in a specific region), there is no diffusion and thus the peak temperatures can be much higher than where thermal diffusion takes place. Taking a typical value of heat conductivity for biological tissue of $0.6 \mathrm{~W} \cdot \mathrm{m}^{-1} \cdot \mathrm{K}$ and cell size of $10 \mu \mathrm{m}$, the heat dissipation time from a single cell within living tissue is on the order of $100 \mu \mathrm{s}$.

To refine the laser parameters (wavelength, spotsize, pulse duration, radiant exposure) appropriate for laser surgery and tissue remodeling as well as diagnostic laser applications, it is important to understand the spatial and temporal patterns of cellular responses to thermal insults up to and including the threshold for irreversible cellular damage or ablation in these short, high temperature pulses.

In a previous study, ${ }^{5}$ we generated an Hsp70-luc reporter gene and used it to study Hsp70 expression patterns in stable lines of NIH3T3 fibroblast cells to determine the dynamic response of Hsp70 expression. Transfected cells were first treated at $42^{\circ} \mathrm{C}$ in a water bath for durations of $20 \mathrm{~min}$ and luciferase expression was monitored at 2, 10, and $24 \mathrm{~h}^{5}$ Shorter exposures to higher temperatures were studied from 50 to $64{ }^{\circ} \mathrm{C}$ for exposure times of 5 to $35 \mathrm{~s}$ using a thermocycler. ${ }^{5}$ In this and other studies, ${ }^{5,6}$ cells treated with the shorter duration thermal stress (several seconds) were found to survive relatively high temperatures $\left(\geqslant 50^{\circ} \mathrm{C}\right)$; however, extremely short durations, corresponding to thermal confinement times (on the order of microseconds) were not evaluated.

1083-3668/2008/13(3)/030501/3/\$25.00 ๑ 2008 SPIE 


\section{JBO LETTERS}

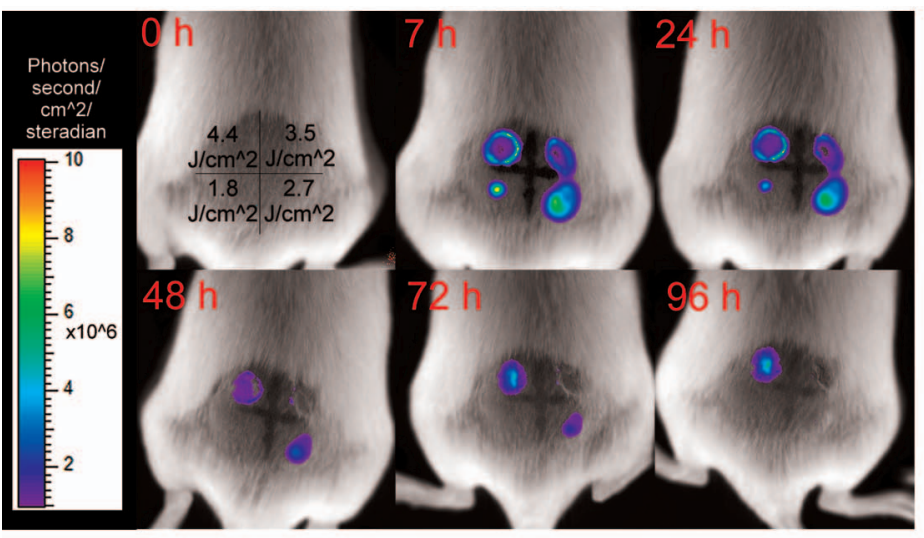

(a)
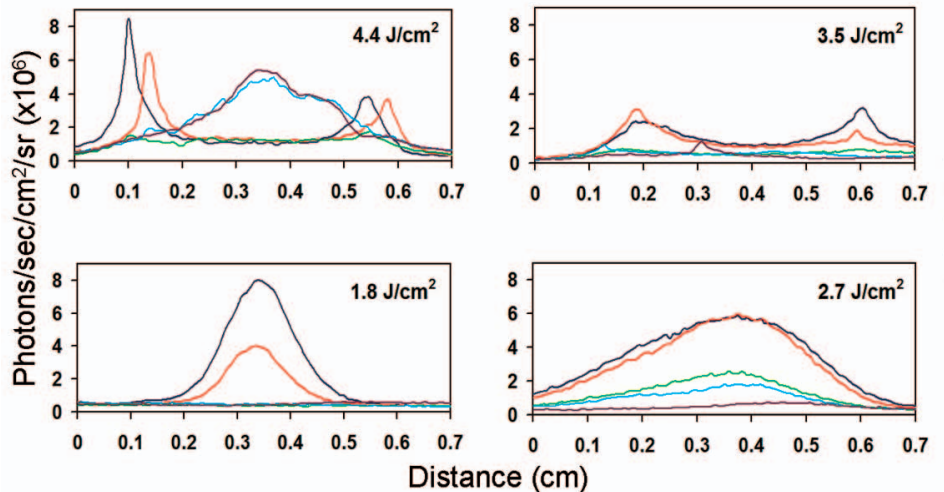

(c)

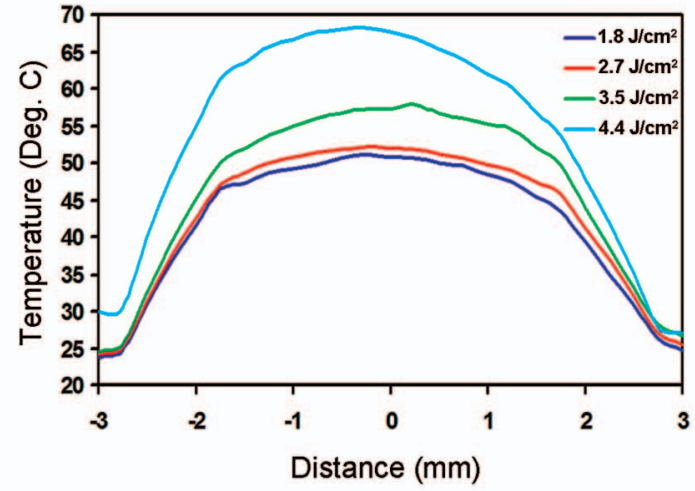

(b)

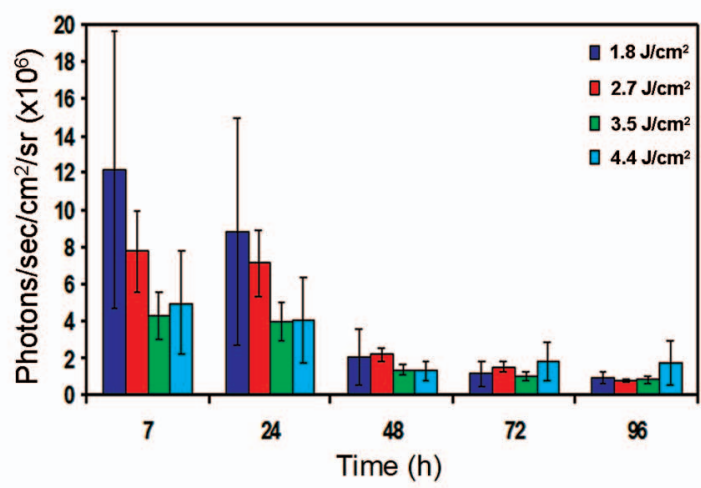

(d)

Fig. 1 Bioluminescent signals indicative of the in vivo thermal response of mouse skin following laser injury. (a) $\mathrm{A} 1-\mathrm{s} \mathrm{CO}_{2}$ laser pulse at $\lambda$ $=10.6 \mu \mathrm{m}$ was used at four different radiant exposures $\left(1.8,2.7,3.5\right.$, and $\left.4.4 \mathrm{~J} / \mathrm{cm}^{2}\right)$. One representative mouse is shown here with images taken at $7,24,48,72$, and $96-\mathrm{h}$ post laser heating. The images are represented with a common false-color scale representing the emitted bioluminescence radiance from $1 \times 10^{6}$ to $1 \times 10^{7}$ photons $/ \mathrm{s} / \mathrm{cm}^{2} /$ steradian. All images were taken ten minutes after IP injection of luciferin. Note the loss of signal for the lowest radiant exposure after $48 \mathrm{~h}$, and the increase for the center region of the highest radiant exposure after $72 \mathrm{~h}$. (b) Each panel consists of a series of 7-mm-long profile plots across the 6-mm laser lesion at each of the five time points and four radiant exposures for the 1-s $\mathrm{CO}_{2}$ laser pulse. A Gaussian pattern of expression is seen for the lowest radiant exposure. At the high radiant exposures, the center region of the pulse is unable to emit light at the early time points. The region immediately surrounding this center region emits light corresponding to Hsp70 expression quite highly at these time points. The surrounding region's expression declines to background levels by the 72-h time point, while the expression in the center region begins to increase between 72 and $96 \mathrm{~h}$. (c) The temperature profile for each laser radiant exposure at the end of the laser pulse is shown for comparison to the bioluminescence expression. The temperature profile was obtained with a thermal camera (A20M Researcher, FLIR Systems, Boston, Massachusetts). (d) The average radiance in photons $/ \mathrm{s} / \mathrm{cm}^{2} /$ steradian of a $7-\mathrm{mm}$-diam region of interest (ROI) around each laser heated spot is shown for the four radiant exposures used in this experiment at 7, 24, 48, 72, and $96 \mathrm{~h}$. Each data point is for $\mathrm{N}=4$ mice and the error bars represent the standard error of the mean (SEM). A general decrease in the bioluminescence and thus the Hsp70 expression is seen over time, while a late recovery in the center region of the highest radiant exposure region is seen at the later time points.

Transgenic $(\mathrm{Tg})$ mice, with the Hsp70 promoter and a reporter gene, have been used in analysis of thermal stress. ${ }^{8}$ To facilitate analysis of thermal stresses at very short pulse durations, we have developed a Tg mouse based on a modified reporter, construct that employs the Hsp70A1 promoter and a dual reporter, comprised of firefly luciferase and enhanced green fluorescent protein (eGFP) joined by the $2 \mathrm{~A}$ "ribosome slippage site" from the foot and mouth disease virus (Hsp70A1-luc-2A-GFP), ${ }^{5,6,9}$ This dual reporter construct allows for the macroscopic analysis of Hsp70 expression in skin in vivo via luciferase expression using BLI. We used this $\mathrm{Tg}$ mouse to assess laser thermal stress and the resultant spatiotemporal patterns of Hsp70 expression in the skin in vivo after irradiation.

\section{Experimental Methods}

\subsection{Transgenic Reporter Mouse}

The Hsp70-luc reporter was described previously, and shown to accurately report levels of Hsp70 transcription in cell lines. ${ }^{5}$ To increase the functional capability of this reporter construct, the gene coding for enhanced green fluorescent protein (eGFP, Clontech, Palo Alto, California) was added. For this, we employed the dual reporter gene L2G (Luc-2AeGFP) consisting of a modified firefly luciferase gene (pGL3, Promega Incorporated, Madison, Wisconsin) joined to 54 base pairs (bp) of the FMDV 2A sequence ${ }^{9}$ and, via $24 \mathrm{bp}$ of polylinker sequence, to the eGFP gene at the $3^{\prime}$ end. Using the restriction enzyme sites Hind III and Not I, the L2G gene was inserted into the vector pcDNA3.1(-) (Invitrogen, Carls- 
bad, California), in which the CMV promoter had been replaced by the Hsp70A1 promoter. ${ }^{5}$ Finally, the Hsp70-L2G reporter gene fragment was excised from the vector backbone and gel purified for pronuclear injection. Microinjection was performed by the Transgenic Core Facility in the Department of Pathology at Stanford University using preimplantation embryos derived from FVB mice. Potential founders were screened by polymerase chain reaction and tested for thermal response by exposure to mild thermal stress and BLI using an IVIS imaging system (Xenogen, Alameda, California). The selected Tg mouse line (FVB.Hsp70-luc-2A-eGFP; short form-Hsp70A1-L2G) was bred to homozygosity, and four to eight week old female mice were used for the in vivo experiments.

\subsection{Laser Thermal Stress Analysis}

Tissue thermal stress was generated with a $100-\mathrm{W} \mathrm{CO}_{2}$ laser (PLX-100, Parallax Technology Incorporated, Waltham, Massachusetts) and evaluated in the Hsp70A1-L2G mouse. Using the $\mathrm{CO}_{2}$ laser, the dorsal skin of the transgenic mice was irradiated at a wavelength of $10.6 \mu \mathrm{m}$. Mice were shaved and depilated $24 \mathrm{~h}$ prior to irradiation with the laser-24 h was sufficient time to prevent residual expression of Hsp70 caused by shaving. Experiments were performed with a 1-s pulse duration. Four irradiation spots were generated at separate locations on the back of each mouse in a grid pattern at 1.8, 2.7, 3.5, and $4.4 \mathrm{~J} / \mathrm{cm}^{2}$ [Fig. 1(a)]. A 6-mm-diam flat-top spot, created by irradiating the mouse with the central part of a 1 -in. Gaussian beam with a $6-\mathrm{mm}$ hole drilled through an aluminum plate, was used to generate an affected area large enough to provide adequate resolution to determine the expression profile using an IVIS 200 imaging system (Xenogen/Caliper Life Sciences), with high resolution binning (4) and a $3.9-\mathrm{cm}$ field of view to obtain a $60-\mu \mathrm{m}$ resolution across each treated location. BLI was performed 10 -min postintraperitoneal (IP) injection of $50-\mu \mathrm{l} / 10-\mathrm{g}$ mouse of $30-\mathrm{mg} / \mathrm{ml}$ stock solution of D-luciferin (Biosynth L-8220, Staad, Switzerland) with a 1-min integration time.

\section{Results}

BLI was used to analyze the approximate levels of Hsp70 transcriptional activation following laser injury to the skin. This approach enabled macroscopic analysis of individual lesions in live mice with temporal resolution that is relevant to Hsp70 induction [Fig. 1(a)]. The short half life of the luciferase enzyme $\left(\sim 2 \mathrm{~h}^{10}\right)$ results in the assay being one of transcriptional activity, ${ }^{5}$ and as such both the activation and cessation of transcription can be assessed. ${ }^{5}$ The levels of Hsp70 and luciferase will differ with protein stability, and the half life of luciferase enzyme results in a dynamic assay., Figure 1 reveals the temporal responses of the heat shock reporter to irradiation of skin with a $\mathrm{CO}_{2}$ laser. Profile plots of each lesion generated by the laser were obtained at five imaging times for comparison [Fig. 1(b)] by placing a 7-mm line across the center of each lesion using ImageJ (National Institutes of Health, Washington, DC) and examining the profile of expression across this line. The profile plots are from the same images used in Fig. 1(a). The peak radiance was obtained by placing a region of interest (ROI) with a 7-mm-diam around each irradiated spot of each mouse image and measuring the peak radiance using Living Image Software (Xenogen/Caliper, Hopkinton, Massachusetts), Fig. 1(d).

\section{Discussion and Conclusions}

Analysis shows a qualitative peak of expression at 7-h postirradiation, which is apparent up to $96 \mathrm{~h}$. Intermediate energy levels caused reduced expression. The results are displayed as a linear profile plots across the center of each lesion. Highest radiant exposure images show a ring-like expression contour surrounding an inner region of reduced expression. This expression contour is seen as two distinct peaks at either edge of the lesion in the profile plots. Since luciferase activity is dependent on the metabolic activity of the cell, reporter activity is lost at the time of cell death and the patterns of expression are consistent with this link to cell viability. Intermediate energy levels produced a Gaussian profile similar to those obtained for the lowest radiant exposures. Antibody staining of GFP in tissue sections from samples subjected to similar laser parameters have indicated that GFP and Hsp70 expression correlate spatially throughout the laser spot (data not shown).

In this study, we demonstrate the creation and validation of a transgenic mouse that enables the rapid analysis of cellular injury and survival in response to laser tissue injury. This Hsp70 reporter mouse model will serve as an excellent indicator of cellular stress, cell survival, collateral damage, and wound healing in vivo.

\section{Acknowledgments}

This work was funded by a grant from the Air Force Office of Scientific Research (F49620-00-1-0349). Contag is a consultant for Caliper Life Sciences, Hopkinton, Massachusetts.

\section{References}

1. C. Soti, E. Nagy, Z. Giricz, L. Vigh, P. Csermely, and P. Ferdinandy, "Heat shock proteins as emerging therapeutic targets," Br. J. Pharmacol. 146, 769-780 (2005).

2. A. G. Pockley, "Heat shock proteins, inflammation, and cardiovascular disease," Circulation 105, 1012-1017 (2002).

3. N. B. Gibbons, R. W. G. Watson, R. N. T. Coffey, H. P. Brady, and J. M. Fitzpatrick, "Heat-shock proteins inhibit induction of prostate cancer cell apoptosis," Prostate 45, 58-65 (2000).

4. R. E. Ferrando, S. T. Schuschereba, J. A. Quong, and P. D. Bowman, "Carbon dioxide laser induction of heat shock protein 70 synthesis: Comparison with high temperature treatment," Lasers Med. Sci. 10, 207-212 (1995).

5. C. E. O'Connell-Rodwell, D. Shriver, D. M. Simanovskii, C. McClure, Y. A. Cao, W. Zhang, M. H. Bachmann, J. T. Beckham, E. D. Jansen, D. Palanker, H. A. Schwettman, and C. H. Contag, "A genetic reporter of thermal stress defines physiologic zones over a defined temperature range," FASEB J. 18, 264-271 (2004)

6. J. T. Beckham, M. A. Mackanos, C. Crooke, T. Takahashi, C. O'Connell-Rodwell, C. H. Contag, and E. D. Jansen, "Assessment of cellular response to thermal laser injury through bioluminescence imaging of heat shock protein 70," Photochem. Photobiol. 79, 76-85 (2004).

7. G. J. Wilmink, S. R. Opalenik, J. T. Beckham, J. M. Davidson, and E. D. Jansen, "Assessing laser-tissue damage with bioluminescent imaging," J. Biomed. Opt. 11, 041114 (2006).

8. E. S. Christians and I. J. Benjamin, "The stress or heat shock (HS) response: insights from transgenic mouse models," Methods 35, 170175 (2005)

9. M. L. Donnelly, L. E. Hughes, G. Luke, H. Mendoza, E. ten Dam, D. Gani, and M. D. Ryan, "The 'cleavage' activities of foot-and-mouth disease virus $2 \mathrm{~A}$ site-directed mutants and naturally occurring ' $2 \mathrm{~A}$ like' sequences," J. Gen. Virol. 82, 1027-1041 (2001).

10. G. M. Leclerc, F. R. Boockfor, W. J. Faught, and L. S. Frawley, "Development of a destabilized firefly luciferase enzyme for measurement of gene expression," BioTechniques 29, 590 (2000). 